\title{
Depression Relationship with Dietary Patterns and Dietary Inflammatory Index in Women: Result from Ravansar Cohort Study
}

This article was published in the following Dove Press journal:

Neuropsychiatric Disease and Treatment

\author{
Jalal Moludi iD ${ }^{1-3}$ \\ Mehdi Moradinazar iD ' \\ Behrooz Hamzeh' \\ Farid Najafi (iD ${ }^{4}$ \\ Davood Soleimani ${ }^{3}$ \\ Yahya Pasdar (iD) ${ }^{1,3}$
}

'Research Center for Environmental Determinants of Health (RCEDH), Kermanshah University of Medical Sciences, Kermanshah, Iran; ${ }^{2}$ Clinical Research Development Center, Imam Reza Hospital, Kermanshah University of Medical Sciences, Kermanshah, Iran; ${ }^{3}$ Department of Nutritional Sciences, School of Nutritional Sciences and Food Technology, Kermanshah University of Medical Sciences, Kermanshah, Iran; ${ }^{4}$ Social Development and Health Promotion Research Center, Kermanshah University of Medical Sciences, Kermanshah, Iran
Correspondence: Yahya Pasdar Department of Nutritional Sciences, School of Nutritional Sciences and Food Technology, Kermanshah University of Medical Sciences, Kermanshah, Iran $\mathrm{Tel}+98-8338362008$

Email Yahya.pasdar@kums.ac.ir
Background and aims: Chronic inflammation is thought to have a major role in the pathophysiology of depression. Diet has been shown to modulate the inflammatory state, thus emphasizing its potential as a therapeutic role in depression. But, little is known about the relationship between dietary intake and depression. The current study aimed to investigate the relationship between major dietary patterns, a dietary inflammatory index (DII) score, and depression among women.

Methods and Materials: This cross-sectional study included 4630 women aged 35-65 years using baseline data from the Ravansar Non-Communicable Diseases (RaNCD) cohort study in Western Iran. Diet was evaluated using a validated 125-item food frequency questionnaire (FFQ) to determine DII scores and dietary patterns. Traditional, healthy, and unhealthy dietary patterns were extracted using factor analyses.

Results: A significant upward trend in the odds of depression was observed across the tertiles s of DII scores (P-trend: 0.019). After the adjustment for possible risk factors, a high adherence to an unhealthy dietary pattern was associated with a higher risk of depression than a low adherence (OR: 1.63; 95\% CI: 1.1-2.4). A high adherence to a healthy dietary pattern was associated with the lower odds (OR: 0.61; 95\% CI: 0.04-0.92). Among the main food groups, a high intake of eggs and refined grains was associated with a higher risk of depression.

Conclusion: In women, a refined grain dietary pattern is a risk factor for depression, whereas a healthy dietary pattern is protective. We have also shown that adherence to a proinflammatory diet was significantly associated with depression. Adherence to a dietary pattern with high intakes of dairy products, seafood, red meats, nuts, vegetables, fruits, flavor, and vegetable oils and diets with low inflammatory properties were associated with a lower risk of depression in women.

Keywords: depression, food group, dietary pattern, dietary inflammatory index

\section{Introduction}

The World Health Organization (WHO) has ranked depression as the most common mental disorder worldwide; it is characterized by tiredness, sadness, and lack of interest in daily life activities. ${ }^{1}$ Depressive disorders are an important cause of disability and the second leading contributor to the global burden of disease. ${ }^{2,3}$ It has been predicted that the lifetime prevalence of a depressive disorder is approximately 2 -fold higher in women (22\%) than men (12\%). Antidepressants attenuate the symptoms of depression, but their efficacy is not always optimal and they are 
often associated with cardiovascular side effects. ${ }^{4,5}$ Consequently, it may be best to prevent depressive disorder in its early stages.

Inflammation also is plays an important role in the pathogenesis of depression. ${ }^{6}$ An inflammatory diet has been found to be associated with the depression. ${ }^{7,8}$ Among various factors that contribute to the setting of an inflammatory milieu in the context of depression, dietary intakes could play a vital role. ${ }^{9}$ Over the past few years' evidence has emerged about the interplay between nutrition and inflammation with mental disorders such as depression. ${ }^{6,10}$ Diet directly affects neurotransmitters, hormones, gut microbiota population, and inflammation, which have a critical role in signal pathways related to sleep, mood, and behavior. ${ }^{7,11}$ Present clinical evidence also supports the view that dietary supplements with anti-inflammatory properties such as omega-3 fatty acids, zinc, and vitamin $\mathrm{D}$ can attenuate the depression symptoms. ${ }^{12-14}$ Nowadays, there is sufficient knowledge of the relationship between individual dietary components and depressive disorders. However, this approach does not take into account many aspects of diet such as the interaction and cumulative effects of nutrients or foods ingested together. ${ }^{9,15}$ The main challenge in understanding the role of diet in the etiology of depression, is the description of this complex exposure. One method is the identification of dietary patterns through statistical modeling such as factor analysis. Recent reviews have shown that healthy dietary patterns are associated with a decreased risk of depression or, ${ }^{16}$ but these are not general findings. ${ }^{17,18,19}$

The Dietary Inflammatory Index (DII) is a relatively new dietary index that is based on peer-reviewed research focusing on diet and inflammation and is standardized to world average dietary intake. ${ }^{20,21}$ The DII was established to offer a means for appraising the total inflammatory latent of the diet. ${ }^{21}$ Formerly, the DII was related to a many of diseases including markers of chronic inflammation, diabetes, and cancer ${ }^{21,22}$. However, there is very limited evidence on the association between dietary patterns and depression. Understanding the role of inflammation in the background of depression will raise the development of therapeutic strategies in order to treat and even prevent the underlying inflammation, thus improving depression outcomes.

As onset and progression of depression is associated with a chronic pro-inflammatory state. ${ }^{6}$ We hypothesized that the higher DII scores, indicating more proinflammatory dietary intakes, are associated with increased depression incidence. There is paucity information about effect of DII score and depression in general population, so the aim of this study was to determine the association between depression and specific food groups and dietary patterns, and also its relationship with the DII in Ravansar Non-Communicable Diseases (RaNCD) cohort study, Kermanshah, Iran.

\section{Methods and Materials Study Design}

A cross-sectional was performed within the framework of the Ravansar Non-Communicable Disease (RaNCD) cohort study. Briefly, RaNCD is a population-based ongoing study designed to determine the results of chronic diseases and death in Ravansar (one of the cities of Kermanshah province in Iran) since 2014. This study is one of the parts of Iran's mega-cohort study PERSIAN (Prospective Epidemiological Research Studies in Iran). Ravansar, a region with urban and rural areas is positioned in western of Iran in the Kermanshah state nearby the Iraq border which has about 50,000 inhabitants with Kurdish ethnicity. The PERSIAN cohort study has been set up in 10 geographical regions in Iran. ${ }^{23}$ These areas have been carefully chosen based on local disease patterns, risk factors, and local mortality and morbidity. The chief attention of clinical researches is on prevention of noncommunicable diseases, which includes about $70 \%$ of the diseases and causes of mortality in the country. ${ }^{24}$

For this study, we used data from the females between the ages of 25 and 65 years. A total of 5289 women answered to the first survey in 2014 . The inclusion criteria for contributor enrolment were as follows: 1) age 25-65 years; 2) at least dwelling of 1 year in Ravansar; 3) willingness to participate; 4) receiving the written consent form. Women were excluded if they reported a history of cardiovascular disease, cancer, thyroid disease and inflammatory disease, and people who were addicted to alcohol and drug abusers. Finally, 4630 subjects were included in this study. All participants provided informed consent based on the Declaration of Helsinki guidelines at the beginning of the measurement. All measurements were performed by well-trained interviewers at the study site in Ravansar.

\section{Outcome}

The study participant's screened for symptomatic or nonsymptomatic depression by expert psychologists. Screening comprised a clinical examination and a self-administered 
questionnaire. Also, information on antidepressants used by a prescriptions were obtained from the participants.

\section{Anthropometric Assessment}

Participants' height was measured using an automatic stadiometer BSM 370 (Biospace Co., Seoul, Korea) with a precision of $0.1 \mathrm{~cm}$ while subjects were in the standing position without shoes. Weight measurements were performed using an InBody 770 device (Inbody Co, Seoul, Korea) with wearing the least clothing and without shoes. Height and weight measurements were performed only once. BMI was calculated based on the following formula: weight $(\mathrm{kg}) /$ height $^{2}$ (meter).

\section{Dietary Assessment}

Nutritional data were collected using the validated national Iranian Food Frequency Questionnaire (FFQ). ${ }^{25}$ This questionnaire includes 125 food items appropriate for the Iranian population including bread, cereal, grains, meat and meat products, milk and dairy products, vegetables, fruits, types of oil and oilseeds, sugar, miscellaneous food products, spices, and food supplements. ${ }^{26}$ Also, some local foods were added to the questionnaire. All participants provided an answer to their frequencies of food intake (daily, weekly, monthly and annually) according to standard portion size for each food item and then each participant's reported intake was transformed to weight using standard Iranian household measures. Dietary information was converted to energy and nutrients using revised Nutritionist IV software [Nutritional Database Manager 4.0.1, Nutritionist IV (First Data Bank, USA), version 3.5.2].

Details of the development and construct validation of the DII have been described elsewhere. ${ }^{21,22}$ Briefly, dietary macronutrients and micronutrients were scored according to whether they are able to increase $(+1)$ or decrease $(-1)$ or have no effect $(0)$ on inflammatory biomarkers. These scores were weighted based on study design. To determine dietary patterns food items were included in predefined main food groups based on their nutritional similarities or culinary usage. ${ }^{27}$ We also considered some specific food items (eggs, salt, and sugar) as separated food groups because of specific nutrition profiles. Fifteen food groups were included in Factor Analysis (Table 1).

\section{Other Assessments}

Experienced interviewers collected demographic information using face to face interview including age, gender, economic status, educational level, marital status; smoking, medical history, and drug history by the validated questionnaire. To assess the physical activity status, the short form of the International Physical Activity Questionnaire (IPAQ) was used. Participants were allocated to one of three groups using daily METs and determined cutoff points with low (24-36.5), moderate (36.6-44.9), and severe ( $\geq 45)$ activity levels.

\section{Statistical Analysis}

All statistical analysis was performed with the SPSS software version 16.0 (SPSS, Inc., Chicago, IL). The distribution of variables was analyzed using the Kolmogorov-Smirnov test. Comparison of demographic characteristics was undertaken using independent-sample t-tests for normal distributed variables, Mann-Whitney $U$-test for non-normal distributed variables, and chi-square test for qualitative variables. The relationship between demographic variables and being depressed was calculated by the spearman test.

We applied Factor Analysis to extract dietary patterns from 15 food groups. The correlation among food groups was determined with the Kaiser-Meyer-Olkin and Bartlett's sphericity test. The number of extracted dietary pattern was decided using an eigenvalue of $>1$ and the shape of scree plot. To make an easier interpretation, retained dietary patterns were rotated using Varimax with Kaiser Normalization method. Food groups of each dietary pattern were determined according to the rotated component matrix of $>0.30$. Dietary patterns were labeled based on the similarity of their food groups with previously wellknown dietary patterns. We used the binary logistic regression to assess the odds of depression with main food groups, dietary patterns, and dietary inflammatory index in the crude model, and energy adjusted model (model 1) and multivariable adjusted model for energy intake, age, BMI, smoking, alcohol abuse, physical activity, and place of living.

\section{Results}

Among 5289 women with completed the food frequency questionnaire, 4630 subjects $(87.5 \%)$ had an energy intake between 800 and $4200 \mathrm{Kcal} /$ days and were included in the final analysis. The demographic characteristics of participants are shown in Table 2. There was a significant association between depression with living place, smoking, body weight, and alcohol consumption.

The relationship between the depression and main food groups is shown in Figure 1. A high intake of eggs, refined grains, and red meats increased the risk of depression, but 
Table I Food Grouping Used in the Dietary Pattern Analyses

\begin{tabular}{|c|c|}
\hline $\begin{array}{l}\text { Food } \\
\text { Groups }\end{array}$ & Food Items \\
\hline $\begin{array}{l}\text { Refined } \\
\text { grains }\end{array}$ & $\begin{array}{l}\text { White bread, rice, potato, flour, macaroni, noodle, } \\
\text { biscuit, cake }\end{array}$ \\
\hline $\begin{array}{l}\text { Whole } \\
\text { grains }\end{array}$ & $\begin{array}{l}\text { Barely bread, whole wheat, wheat germ, oat, bulgur, } \\
\text { corn flakes }\end{array}$ \\
\hline Red meat & $\begin{array}{l}\text { Beef, lamb, camel, sausages, hamburger, processed } \\
\text { meats, organ meats }\end{array}$ \\
\hline Poultry & Chicken, turkey, ostrich \\
\hline seafood & fish, shellfish, seaweed \\
\hline $\begin{array}{l}\text { Dairy } \\
\text { products }\end{array}$ & $\begin{array}{l}\text { Yogurt, dough (yoghurt drink), milk, cheese, curd, } \\
\text { cream, butter, ice cream, pizza cheese }\end{array}$ \\
\hline Legumes & Beans, chickpeas, lima beans, broad beans, lentil, soy \\
\hline Nuts & $\begin{array}{l}\text { Peanut, almond, pistachio, walnut, hazelnut, roasted } \\
\text { seeds }\end{array}$ \\
\hline Eggs & Eggs \\
\hline Vegetables & $\begin{array}{l}\text { Cucumber, tomato, spinach, pepper, mushroom, garlic, } \\
\text { mushroom, carrot, onions, mixed vegetable, lettuce, } \\
\text { cabbage, eggplant, celery, green peas, green beans, } \\
\text { turnip, corn, tomato paste }\end{array}$ \\
\hline Fruits & $\begin{array}{l}\text { Apple, cherries, grapefruit, apricots, plum, kiwi, } \\
\text { strawberry, oranges, grapes, berries, date, barberry, } \\
\text { banana, pomegranate, melon, tangerine, lemon, raisin, } \\
\text { mulberry, persimmon, peach, cantaloupe, watermelon, } \\
\text { pear, fruit juices }\end{array}$ \\
\hline Sugars & Jam, honey, candy, sugar, chocolate, pastry \\
\hline Flavor & Garlic powder, turmeric, ginger, pepper, curry powder \\
\hline $\begin{array}{l}\text { Vegetables } \\
\text { oils }\end{array}$ & Vegetables oils \\
\hline Salt & Salt \\
\hline
\end{tabular}

this association disappeared for red meats after controlling the effect of potential risk factors. Three major dietary patterns were extracted using Factor Analysis method which explained $35.7 \%$ of the total dietary variance with the Kaiser-Meyer- Olkin measure of sampling adequacy of 0.78 and Bartlett's sphericity test $<0001$. The main food groups of each dietary pattern are shown in Table 3 . The first dietary pattern (healthy) explained $16.2 \%$, the second dietary pattern (traditional) explained $10.2 \%$, and the third dietary pattern (Unhealthy) explained $9.3 \%$ of the total dietary variance.
Table 2 Comparison of Demographic Data Between Women with Depression Vs Women Without Depression

\begin{tabular}{|l|l|l|l|}
\hline Variables & $\begin{array}{l}\text { Non-Depressive } \\
\text { Women N=4355 }\end{array}$ & $\begin{array}{l}\text { Depressive } \\
\text { Women } \mathbf{N = 2 7 5}\end{array}$ & P value \\
\hline Age, years & $48.8 \pm 8.5$ & $47.9 \pm 8$ & 0.11 \\
\hline Weight, $\mathrm{Kg}$ & $68.9 \pm 12.8$ & $71.39 \pm 1 \mathrm{I} .3$ & 0.02 \\
\hline BMI, kg/m² & $29.2 \pm 3.7$ & $28.2 \pm 4.5$ & 0.71 \\
\hline WC, cm & $98.1 \pm 10.85$ & $98 \pm 10.56$ & 0.88 \\
\hline HC, cm & $103.4 \pm 9.08$ & $103.8 \pm 8.5$ & 0.45 \\
\hline WHR & $0.95 \pm 0.058$ & $0.94 \pm 0.057$ & 0.15 \\
\hline $\begin{array}{l}\text { MET, min/ } \\
\text { day }\end{array}$ & $39.3 \pm 4.6$ & $38.7 \pm 6.7$ & 0.08 \\
\hline Smoker, $\mathrm{n} \%$ & 5.3 & 16.5 & 0.01 \\
\hline $\begin{array}{l}\text { Alcohol } \\
\text { abuse, } \mathrm{n} \%\end{array}$ & 0.1 & 3.6 & $0.01 *$ \\
\hline $\begin{array}{l}\text { Urban } \\
\text { dwellers, } \mathrm{n} \\
\%\end{array}$ & 58.8 & 64.7 & 0.001 \\
\hline Notes: & & & \\
\hline
\end{tabular}

Notes: Data are presented as mean and standard deviation or frequencies. P-values were extracted from independent-sample $t$-test (quantitative variables) and chisquare test or Fisher's exact test *(qualitative variables).

Abbreviations: BMI, body mass index; WC, waist circumference; HC, hip circumference; WHR, waist/hip ratio; MET, metabolic equivalent.

The odds of having depression across the tertiles of dietary pattern scores is shown in Table 4. A higher adherence to the healthy dietary pattern had no relationship with being the depression in the crude model (P-trend: 0.28) and energy-adjusted model (P-trend: 0.074). However, a significant association and negative relationship appeared after the further adjustment for other risk factors in model 2 (P-trend: 0.039). Women in the highest tertiles of adherence to the traditional dietary pattern had higher risk of depression than those in the lowest tertiles in the crude model (Odds Ratio: 1.75; $95 \%$ CI: 1.22 to 2.25 ) and energy adjusted model (Odds Ratio: 1.68; 95\% CI: 1.13 to 2.51). After further adjustment for other risk factors in model 2, although the women with the higher adherence to traditional dietary pattern had a higher risk of depression compared with lower adherence (Odds Ratio: 1.66; 95\% CI: 1.10 to 2.51), there was no significant upward trend in the odds of having depression with increasing the adherence to this pattern (P-trend: 0.24). In the unhealthy dietary pattern, women who were in the highest tertiles $\mathrm{s}$ had a higher odds of depression compared to those in the 


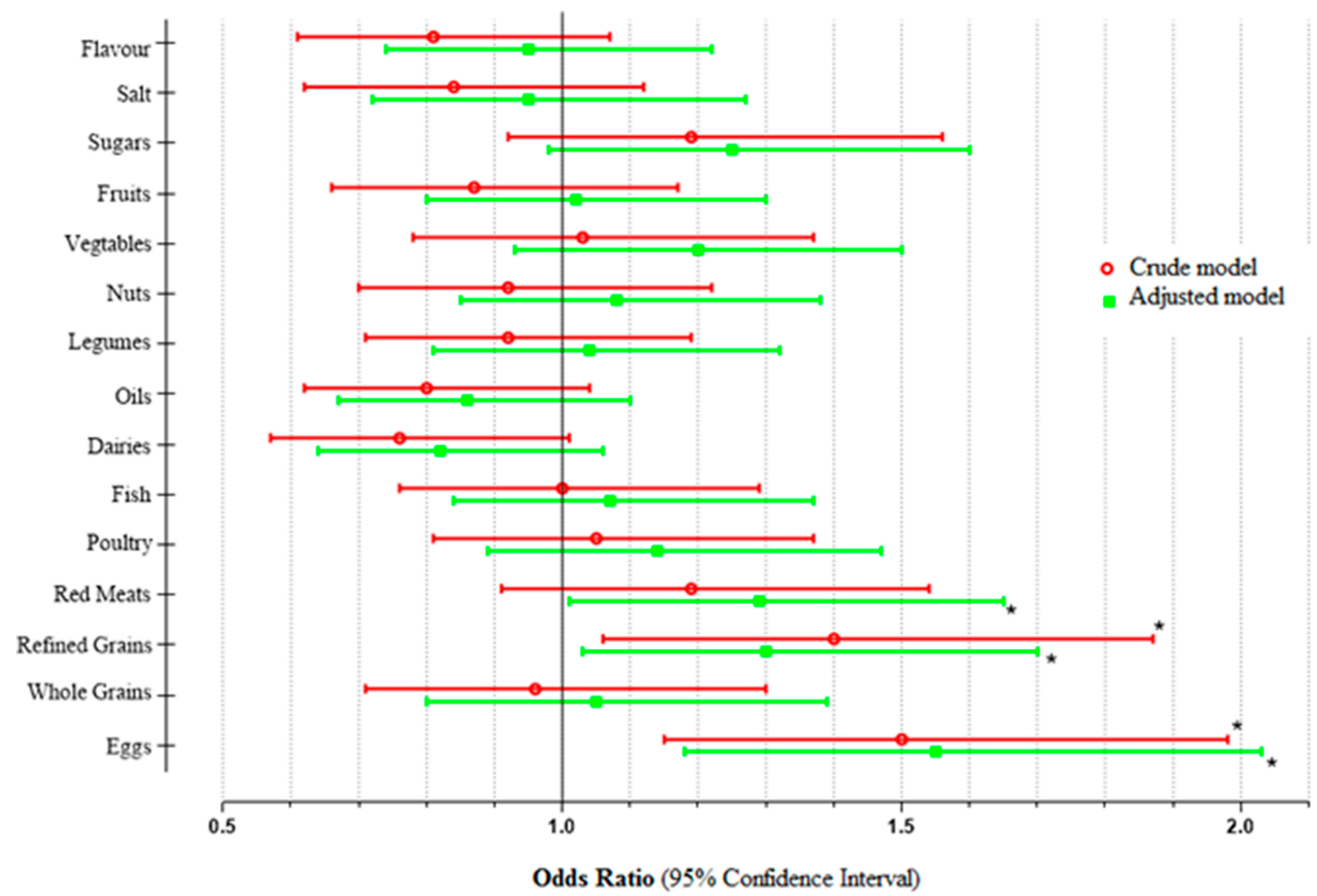

Figure I Crude and adjusted odds ratio for depression across median of the main food groups. Adjusted for age, alcohol abuse, physical activity, smoking, energy intake and place of living $* P$ value was less than 0.05 .

lowest tertiles s even after eliminating the effect of risk factors. We also observed a significant upward trend in the odds of being depression with increasing the adherence to the unhealthy dietary pattern in the crud model (P-trend: 0.009), energy-adjusted model (P-trend: 0.034), and multivariable-adjusted model (P-trend: $0.026)$.

The odds of having depression across the tertiles $\mathrm{s}$ of the DII is shown in Table 5. There was a significant upward trend in the odds of being depression with increasing in the tertiles of DII (P-trend: 0.01). However, this relationship disappeared after the adjustment for energy intake (P-trend: 0.41). With the following further adjustment for other potential risk factors (Model 2), we observed that subjects in the highest tertiles of the dietary inflammatory index $(0.114<)$ had more risk of being depression than subjects in the lowest tertiles s (Odds ratio: $1.47 ; 95 \% \mathrm{CI}$ : 1.07 to 2.03 ; P-value: 0.18 ). Meanwhile, there was a significant upward trend in the odds of having depression across the tertiles of the dietary inflammatory index (P-trend: 0.01).

\section{Discussion}

The present study investigated the relationship of different aspects of diets, including dietary patterns, food groups, and dietary inflammatory potential (as indexes by DII) with depression. Three major dietary patterns were extracted from the data in the current study; including healthy, traditional, and unhealthy diets. The study confirmed that adherence to the healthy pattern was inversely associated with the risk of being depression, while the unhealthy pattern showed a positive association. Among the main food groups, the high intake of eggs and refined grains showed a destructive role in depression independent of potential risk factors. Our finding also revealed that high DII scores were associated with an increasing risk of depressive disorders. These associations could be explained by diet-related factors. Diets containing high added sugar, refined grains, and trans fatty acids can 
Table 3 Rotated Component Matrix of Major Dietary Patterns Identified in Women

\begin{tabular}{|c|c|c|c|}
\hline $\begin{array}{l}\text { Food } \\
\text { Groups }\end{array}$ & $\begin{array}{l}\text { Healthy } \\
\text { Pattern }\end{array}$ & $\begin{array}{l}\text { Traditional } \\
\text { Pattern }\end{array}$ & $\begin{array}{l}\text { Unhealthy } \\
\text { Pattern }\end{array}$ \\
\hline $\begin{array}{l}\text { Dairy } \\
\text { products }\end{array}$ & 0.310 & & \\
\hline Red meats & 0.494 & & \\
\hline Poultry & & 0.366 & \\
\hline Seafood & 0.429 & & \\
\hline $\begin{array}{l}\text { Refined } \\
\text { grains }\end{array}$ & & & 0.604 \\
\hline $\begin{array}{l}\text { Whole } \\
\text { grains }\end{array}$ & & 0.431 & $-0.34 I$ \\
\hline Eggs & & 0.637 & \\
\hline Legumes & & 0.602 & \\
\hline Nuts & 0.670 & & \\
\hline Vegetables & 0.468 & 0.500 & \\
\hline Fruits & 0.648 & & \\
\hline Sugars & & & 0.520 \\
\hline Salt & & & 0.553 \\
\hline Flavor & 0.527 & & \\
\hline $\begin{array}{l}\text { Vegetable } \\
\text { oils }\end{array}$ & 0.605 & & 0.297 \\
\hline
\end{tabular}

Notes: Absolute values less than 0.2 are not displayed for simplicity. Numbers in bold indicated main food groups with significant correlation $(>0.3)$ within each dietary pattern.

cause inflammation and resulting depression. As previous studies have shown that healthy diets such as the Mediterranean or the healthy pattern in our study are associated with a lower incidence of depression. Our study additional supports these results suggesting that healthy diets (low sugar added and high fish intake) are probably

Table 5 Odds Ratio $(95 \% \mathrm{Cl}) *$ for Depression Across the Tertiles ( $\mathrm{T}$ ) of Dietary Inflammatory Index

\begin{tabular}{|l|l|l|l|l|}
\hline Model & TI & T2 & T3 & P-Trend \\
\hline Crude & Ref. & I.23 (0.89-I.69) & I.48 (I.I-I.99) & 0.010 \\
Model I & Ref. & I.13(0.82I-I.58) & I.I8 (0.78-1.79) & 0.419 \\
Model 2 & Ref. & $1.27(0.92-1.75)$ & $1.47(1.07-2.03)$ & 0.019 \\
\hline
\end{tabular}

Notes: *Odds ratios $(95 \% \mathrm{Cl})$ were obtained using binary logistic regression. Model I: adjusted for energy intake. Model 2: model I plus the further adjustment for age, BMI, smoking, alcohol abuse, physical activity, and place of living.
Table 4 Odds Ratio $(95 \% \mathrm{Cl}) *$ for Being Depression Across Tertiles $(\mathrm{T})$ of Dietary Pattern Scores

\begin{tabular}{|c|c|c|c|c|}
\hline $\begin{array}{l}\text { Dietary } \\
\text { Pattern }\end{array}$ & TI & T2 & T3 & P-Trend \\
\hline \multicolumn{5}{|l|}{$\begin{array}{l}\text { Healthy } \\
\text { pattern }\end{array}$} \\
\hline Crude & Ref. & $0.76(0.54-1.07)$ & $0.89(0.64-1.24)$ & 0.28 \\
\hline Model I & Ref. & $0.68(0.48-0.97)$ & $0.7(0.48-1.03)$ & 0.074 \\
\hline Model 2 & Ref. & $0.67(0.46-0.46)$ & $0.61(0.4-0.92)$ & 0.039 \\
\hline \multicolumn{5}{|l|}{$\begin{array}{l}\text { Traditional } \\
\text { pattern }\end{array}$} \\
\hline Crude & Ref. & $1.62(1.12-2.34)$ & $1.75(1.22-2.5 \mathrm{I})$ & 0.006 \\
\hline Model I & Ref. & $1.59(1.09-2.32)$ & $1.68(1.13-2.51)$ & 0.024 \\
\hline Model 2 & Ref. & $1.66(1.13-2.46)$ & $1.66(1.10-2.51)$ & 0.21 \\
\hline \multicolumn{5}{|l|}{$\begin{array}{l}\text { Unhealthy } \\
\text { pattern }\end{array}$} \\
\hline Crude & Ref. & I.II (0.77-I.60) & $1.63(1.16-2.29)$ & 0.009 \\
\hline Model I & Ref. & $1.09(0.75-1.58)$ & $1.55(1.08-2.23)$ & 0.034 \\
\hline Model 2 & Ref. & $1.12(0.77-1.65)$ & $1.63(1.11-2.4 I)$ & 0.026 \\
\hline
\end{tabular}

Notes: *Odds ratios $(95 \% \mathrm{Cl})$ were obtained using binary logistic regression. Model I: adjusted for energy intake. Model 2: model I plus the further adjustment for age, BMI, smoking, alcohol abuse, physical activity, and place of living.

necessary for the prevention of depression. Focusing on the whole dietary intake with calculating DII score which covers many aspects of diet into consideration more exactly shows the relationship between diet and the risk of depression. DII is based in former data of associations and their possible physiological pathways, and each component of the index has already received attention in trying to explain the biological plausibility of its association with inflammation. Recently, an empirical dietary inflammatory index (EDII) has been established using prospective data. ${ }^{28}$ Quite the reverse, EDII is a food-based index, in particular based on food groups. Indices of this kind are more appropriate for clinical use but they are more difficult to be applied across countries and cultures because food components and their relevance in the diet may vary substantially. Therefore, DII seems to capture better the underlying association than EDII in which only food groups are considered.

The unhealthy dietary pattern included a high intake of salt, refined grains, and sugar. Women with a greater adherence to the unhealthy dietary pattern experienced a $63 \%$ amplified risk of depression (OR: 1.63; 95\% CI: 1.11-2.41), with a statically significant $(\mathrm{P}=0.026)$ trend across tertiles s. It is well documented that soft drinks, refined grains, and red meat are associated with the occurrence of depression. ${ }^{8,29}$ Among these are, we also found that refined grains were associated with a significantly 
increased risk of depression. This finding is consistent with previous observations from prospective studies in many cohort studies. ${ }^{30,31}$ Recent meta-analysis resulted that high sugar-sweetened beverages intake is significantly associated with the high risk for depression. ${ }^{30} \mathrm{~A}$ Western dietary pattern, as an unhealthy pattern with proinflammatory properties, promotes inflammation which in turn can lead to depression. In addition, the high intake of sugar may increase the risk of depression by the effect on Brain-Derived Neurotropic Factor (BDNF) levels and inflammation which are both play an important role in depression. ${ }^{32}$ Furthermore, high sugar intakes could induce hypoglycemia through an exaggerated insulin response and whereby impact on hormone levels and hypothetically depression symptoms. ${ }^{33}$ By comparing the results from Afsar, et al, we hope to determine that the higher intake of salt was associated with an increased risk of depression. ${ }^{34} \mathrm{~A}$ high salt intake was included in the unhealthy diet in our study and increased the risk of depression. Salt is the main source of sodium $\left(\mathrm{Na}^{+}\right)$and chloride $\left(\mathrm{Cl}^{-}\right)$intake that plays a major role in pathophysiological conditions. ${ }^{35}$ High salt intake is one of the major causes of hypertension, cardiovascular disease and depression. Salt may be the induction of chronic inflammatory responses through expansion of the $\mathrm{T}$ helper-17 pathway and their inflammatory cytokines. ${ }^{36}$ Taken together, we hypothesize that high intake of sugar, salt, and refined grains have a synergistic effect on depressive.

The healthy dietary pattern in the current study contained a high intake of dairies, nuts, seafood, red meat, fruits, vegetables, flavor, and vegetable oils. Adherence to the healthy pattern was accompanied with a low risk of depression. This association might be attributed to the healthy lifestyle factors related to this pattern. However, this association continued statically significant after adjustment for possible confounders, such as age, BMI, smoking, alcohol abuse, physical activity, and place of living. In agreement with our findings, $\mathrm{Li}$ et al revealed that adherence to a healthy dietary pattern (high intakes of fruit, vegetables, fish, whole grain, olive oil, low-fat dairy and antioxidants and low intakes of animal foods) associated with a decreased risk of depression. ${ }^{37}$ These foods include phytosterols, polyphenols, and omega-3 fatty which through antioxidant and anti-inflammatory properties could downregulate chronic inflammation. Our results also are in agreement with former studies suggesting the fish consumption association with decrease depression. ${ }^{38}$ Omega-3 polyunsaturated fatty acids ( $\omega-3$ PUFA), such as eicosapentaenoic acid (EPA) and docosahexaenoic acid (DHA), have been claimed to produce beneficial effects on depression. ${ }^{13}$

The traditional dietary pattern in our study included a high intake of whole grains, legumes, poultry, eggs, and vegetables was not associated with depression. But, more egg consumption associated with increased risk of depression. Eggs are a rich sources of cholesterol which may be roles in depression. This is inconsistent with what has been found previously, which shows that egg is a good source of high-quality amino acids and rich in vitamins, which these components have been implicated to play a role in reducing the risk of depression. It's may also relate to socioeconomic situation of egg consumers, because of low price of egg compare too other sources of animal proteins. This result can be associated with the natural complexity of egg which is rich in both healthy and unhealthy nutrients. ${ }^{39}$ It is therefore of interest to know whether egg increased risk of depression or not.

Our findings also revealed that a high intake of inflammatory diet (indicated by higher DII scores) was associated with increasing the incidence of depressive. These results possibly indicate that pro-inflammatory diets independently contribute to the onset of depression.

Yahya.pasdar@kums.ac.irThe present study explore the relationship between the dietary pattern and risk of depression from a comprehensive perspective among Iranian women population. Despite its strengths including the use of large data from the cohort study, our study had some limitations. First, we did not measure inflammatory biomarkers. Second, the factor analysis was applied with numerous accidental choices such as food grouping, the number of retained factors, type of rotation, and the naming of factors. However, it is important to note that factor analysis has been verified as an appropriate and suitable way in the extraction of dietary patterns for studying the diet-disease association.

\section{Conclusion}

The current study showed that the adherence to the healthy dietary pattern which rich in a high intake of dairy products, nuts, seafood, red meat, fruits, vegetables, flavor, and vegetable oils might be helpful in the nutritional strategies against depression. Additionally, the intake of eggs and refined grains showed a negative role in depression. We also establish that high DII scores were associated with the high incidence of depressive disorder. These associations could be considered in future studies and in the nutritional strategies and national food policy against depression. 


\section{Data Sharing Statement}

Data will be available upon request from the corresponding author.

\section{Acknowledgments}

The authors would like to thank the Clinical Research Development Center of Imam Reza Hospital, Kermanshah University of Medical Sciences, Kermanshah, Iran for their wise pieces of advice.

\section{Funding}

This study is part of the PERSIAN (Prospective Epidemiological Research Studies in Iran) mega cohort study that was approved by ethics committees at the Ministry of Health and Medical Education, the Digestive Diseases Research Institute, Tehran, Iran. Also, all experiments were performed in accordance with relevant guidelines and regulations. Written informed consent were obtained from each study participant at the beginning of the measurement RaNCD is part of the PERSIAN national cohort, and we would like to thank Prof. Reza Malekzadeh, the Deputy of Research and Technology at the Ministry of Health and Medical Education of Iran and Director of the PERSIAN cohort, as well as Dr. Hossein Poustchi, the Executive Director of the PERSIAN cohort, for all their support during the design and implementation of the RaNCD cohort. This study was supported by the Ministry of Health and Medical Education of Iran and Kermanshah University of Medical Sciences (Grant No. 92472).

\section{Disclosure}

The authors declare that they have no conflict of interest.

\section{References}

1. Baxter A, Scott K, Vos T, Whiteford H. Global prevalence of anxiety disorders: a systematic review and meta-regression. Psychol Med. 2013;43(5):897-910. doi:10.1017/S003329171200147X

2. Vigo D, Thornicroft G, Atun R. Estimating the true global burden of mental illness. Lancet Psychiatry. 2016;3(2):171-178. doi:10.1016/ S2215-0366(15)00505-2

3. Reddy M. Depression: the disorder and the burden. Indian J Psychol Med. 2010;32(1):1. doi:10.4103/0253-7176.70510

4. Predictable S, Laurencic G, Malone DA. Side effects of antidepressants: an overview. Cleveland Clin J Med. 2006;73(4):351. doi:10.3949/ccjm.73.4.351

5. Hengartner MP, Angst J, Rössler W. Antidepressant use prospectively relates to a poorer long-term outcome of depression: results from a prospective community cohort study over 30 years. Psychother Psychosom. 2018;87(3):181-183. doi:10.1159/000488802
6. Berk M, Williams LJ, Jacka FN, et al. So depression is an inflammatory disease, but where does the inflammation come from? BMC Med. 2013;11(1):200. doi:10.1186/1741-7015-11-200

7. Lang UE, Beglinger C, Schweinfurth N, Walter M, Borgwardt S. Nutritional aspects of depression. Cellular Physiol Biochem. 2015;37 (3):1029-1043. doi:10.1159/000430229

8. Bodnar LM, Wisner KL. Nutrition and depression: implications for improving mental health among childbearing-aged women. Biol Psychiatry. 2005;58(9):679-685. doi:10.1016/j.biopsych.2005.05.009

9. Neuhouser ML. The importance of healthy dietary patterns in chronic disease prevention. Nutr Res. 2019;70:3-6. doi:10.1016/j. nutres.2018.06.002

10. Leonard BE. Inflammation and depression: a causal or coincidental link to the pathophysiology? Acta Neuropsychiatr. 2018;30(1):1-16. doi:10.1017/neu.2016.69

11. Koopman M, El Aidy S. Depressed gut? The microbiota-dietinflammation trialogue in depression. Curr Opin Psychiatry. 2017;30(5):369-377. doi:10.1097/YCO.0000000000000350

12. Bahrami A, Mazloum SR, Maghsoudi S, et al. High dose vitamin D supplementation is associated with a reduction in depression score among adolescent girls: a nine-week follow-up study. J Diet Suppl. 2018;15(2):173-182. doi:10.1080/19390211.2017.1334736

13. Godos J, Castellano S, Galvano F, Grosso G. Linking Omega-3 Fatty Acids and Depression. Omega Fatty Acids in Brain and Neurological Health. Elsevier; 2019:199-212.

14. Wang J, Um P, Dickerman BA, Liu J. Zinc, magnesium, selenium and depression: a review of the evidence, potential mechanisms and implications. Nutrients. 2018;10(5):584. doi:10.3390/nu10050584

15. Jacques PF, Tucker KL. Are Dietary Patterns Useful for Understanding the Role of Diet in Chronic Disease? Oxford University Press; 2001.

16. Lassale C, Batty GD, Baghdadli A, et al. Healthy dietary indices and risk of depressive outcomes: a systematic review and meta-analysis of observational studies. Mol Psychiatry. 2019;24(7):965-986. doi:10.1038/s41380-018-0237-8

17. Molendijk M, Molero P, Sánchez-Pedreño FO, Van der Does W, Martínez-González MA. Diet quality and depression risk: a systematic review and dose-response meta-analysis of prospective studies. $J$ Affect Disord. 2018;226:346-354. doi:10.1016/j. jad.2017.09.022

18. Dantzer R. Depression and inflammation: an intricate relationship. Biol Psychiatry. 2012;71(1):4-5. doi:10.1016/j.biopsych.2011.10.025

19. Lopresti AL, Maker GL, Hood SD, Drummond PD. A review of peripheral biomarkers in major depression: the potential of inflammatory and oxidative stress biomarkers. Prog Neuropsychopharmacol Biol Psychiatry. 2014;48:102-111. doi:10.1016/j.pnpbp.2013.09.017

20. Vermeulen E, Brouwer IA, Stronks K, et al. Inflammatory dietary patterns and depressive symptoms in Italian older adults. Brain Behav Immun. 2018;67:290-298. doi:10.1016/j.bbi.2017.09.005

21. Shivappa N, Prizment AE, Blair CK, Jacobs DR, Steck SE, Hébert JR. Dietary inflammatory index and risk of colorectal cancer in the Iowa Women's Health Study. Cancer Epidemiol Prev Biomarkers. 2014;23(11):2383-2392. doi:10.1158/1055-9965.EPI14-0537

22. Shivappa N, Bosetti C, Zucchetto A, et al. Association between dietary inflammatory index and prostate cancer among Italian men. Br J Nutr. 2015;113(2):278-283. doi:10.1017/S0007114514003572

23. Poustchi H, Eghtesad S, Kamangar F, et al. Prospective epidemiological research studies in Iran (the PERSIAN Cohort Study): rationale, objectives, and design. Am J Epidemiol. 2018;187(4):647-655. doi:10.1093/aje/kwx314

24. Pasdar Y, Najafi F, Moradinazar M, et al. Cohort profile: Ravansar Non-Communicable Disease cohort study: the first cohort study in a Kurdish population. Int $J$ Epidemiol. 2019;48(3):682-3f. doi:10.1093/ije/dyy296 
25. Moradi S, Pasdar Y, Hamzeh B, et al. Comparison of 3 nutritional questionnaires to determine energy intake accuracy in Iranian adults. Clin Nutr Res. 2018;7(3):213-222. doi:10.7762/cnr.2018.7.3.213

26. Mirmiran P, Esfahani FH, Mehrabi Y, Hedayati M, Azizi F. Reliability and relative validity of an FFQ for nutrients in the Tehran lipid and glucose study. Public Health Nutr. 2010;13 (5):654-662. doi:10.1017/S1368980009991698

27. Soleimani D, Ranjbar G, Rezvani R, Goshayeshi L, Razmpour F, Nematy M. Dietary patterns in relation to hepatic fibrosis among patients with nonalcoholic fatty liver disease. Diabetes Metab Syndrome Obesity. 2019;12:315. doi:10.2147/DMSO.S198744

28. Tabung FK, Smith-Warner SA, Chavarro JE, et al. Development and validation of an empirical dietary inflammatory index. J Nutr. 2016;146(8):1560-1570. doi:10.3945/jn.115.228718

29. Gangwisch JE, Hale L, Garcia L, et al. High glycemic index diet as a risk factor for depression: analyses from the Women's Health Initiative. Am J Clin Nutr. 2015;102(2):454 463. doi:10.3945/ajcn.114.103846

30. Hu D, Cheng L, Jiang W. Sugar-sweetened beverages consumption and the risk of depression: a meta-analysis of observational studies. J Affect Disord. 2019;245:348-355. doi:10.1016/j.jad.2018.11.015

31. Knüppel A, Shipley MJ, Llewellyn CH, Brunner EJ. Sugar intake from sweet food and beverages, common mental disorder and depression: prospective findings from the Whitehall II study. Sci Rep. 2017;7(1):6287. doi:10.1038/s41598-017-05649-7

32. Beilharz J, Maniam J, Morris M. Short-term exposure to a diet high in fat and sugar, or liquid sugar, selectively impairs hippocampal-dependent memory, with differential impacts on inflammation. Behav Brain Res. 2016;306:1-7. doi:10.1016/j.bbr.2016.03.018
33. Everson-Rose SA, Meyer PM, Powell LH, et al. Depressive symptoms, insulin resistance, and risk of diabetes in women at midlife. Diabetes Care. 2004;27(12):2856-2862. doi:10.2337/diacare.27.12.2856

34. Afsar B. The relationship between cognitive function, depressive behaviour and sleep quality with 24-h urinary sodium excretion in patients with essential hypertension. High Blood Pressure Cardiovasc Prev. 2013;20(1):19-24. doi:10.1007/s40292-013-0002-7

35. Suhail M. Na+, K+-ATPase: ubiquitous multifunctional transmembrane protein and its relevance to various pathophysiological conditions. J Clin Med Res. 2010;2(1):1.

36. Kleinewietfeld M, Manzel A, Titze J, et al. Sodium chloride drives autoimmune disease by the induction of pathogenic T H 17 cells. Nature. 2013;496(7446):518. doi:10.1038/nature11868

37. Li Y, Lv M-R, Wei Y-J, et al. Dietary patterns and depression risk: a meta-analysis. Psychiatry Res. 2017;253:373-382. doi:10.1016/j. psychres.2017.04.020

38. Nemets H, Nemets B, Apter A, Bracha Z, Belmaker R. Omega-3 treatment of childhood depression: a controlled, double-blind pilot study. Am J Psychiatry. 2006;163(6):1098-1100. doi:10.1176/ ajp.2006.163.6.1098

39. Tsai AC, Chang T-L, Chi S-H. Frequent consumption of vegetables predicts lower risk of depression in older Taiwanese-results of a prospective population-based study. Public Health Nutr. 2012;15 (6):1087-1092. doi:10.1017/S1368980011002977
Neuropsychiatric Disease and Treatment

\section{Publish your work in this journal}

Neuropsychiatric Disease and Treatment is an international, peerreviewed journal of clinical therapeutics and pharmacology focusing on concise rapid reporting of clinical or pre-clinical studies on a range of neuropsychiatric and neurological disorders. This journal is indexed on PubMed Central, the 'PsycINFO' database and CAS, and is the official journal of The International Neuropsychiatric Association (INA). The manuscript management system is completely online and includes a very quick and fair peer-review system, which is all easy to use. Visit http://www.dovepress.com/testimonials.php to read real quotes from published authors. 